\section{Reduced frequencies and functional impairment of dendritic cell subsets and non-classical monocytes in myelodysplastic syndromes}

Nathalie van Leeuwen-Kerkhoff, ${ }^{1}$ Theresia M. Westers, ${ }^{1}$ Pino J. Poddighe ${ }^{2}$ Giovanni A.M. Povoleri, ${ }^{3}$ Jessica A. Timms, ${ }^{4}$ Shahram Kordasti, ${ }^{4,5 \#}$ Tanja D. de Gruijl ${ }^{6 \#}$ and Arjan A. van de Loosdrecht ${ }^{1 \#}$

${ }^{1}$ Department of Hematology, Amsterdam University Medical Centers, Vrije Universiteit Amsterdam, Cancer Center Amsterdam, the Netherlands; ${ }^{2}$ Department of Clinical Genetics, Amsterdam University Medical Centers, Vrije Universiteit Amsterdam, the Netherlands; ${ }^{3}$ Department Inflammation Biology, King's College London, Center for Inflammation Biology and Cancer Immunology, London, UK; ${ }^{4}$ Systems Cancer Immunology Lab, Comprehensive Cancer Center, King's College London, London, UK; ${ }^{5}$ Dipartimento Scienze Cliniche e Molecolari, UNIVPM, Ancona, Italy and ${ }^{6}$ Department of Medical Oncology, Amsterdam University Medical Centers, Vrije Universiteit Amsterdam, Cancer Center Amsterdam, the Netherlands

${ }^{\#}$ SK, TDG and AAL contributed equally as co-senior authors.

\section{ABSTRACT}

I n myelodysplastic syndromes (MDS) the immune system is involved in pathogenesis as well as in disease progression. Dendritic cells (DC) Lare key players of the immune system by serving as regulators of immune responses. Their function has been scarcely studied in MDS and most of the reported studies didn't investigate naturally occurring DC subsets. Therefore, we here examined the frequency and function of DC subsets and slan+ non-classical monocytes in various MDS risk groups. Frequencies of DC as well as of slan+ monocytes were decreased in MDS bone marrow compared to normal bone marrow samples. Transcriptional profiling revealed down-regulation of transcripts related to pro-inflammatory pathways in MDS-derived cells as compared to normal bone marrow. Additionally, their capacity to induce T-cell proliferation was impaired. Multidimensional mass cytometry showed that whereas healthy donor-derived slant monocytes supported Th1/Th17/Treg differentiation/expansion their MDS-derived counterparts also mediated substantial Th2 expansion. Our findings point to a role for an impaired ability of DC subsets to adequately respond to cellular stress and DNA damage in the immune escape and progression of MDS. As such, it paves the way toward potential novel immunotherapeutic interventions.

\section{Introduction}

Development of human dendritic cells (DC) occurs in the bone marrow (BM), where they originate from common precursor cells and differentiate into specialized subsets: plasmacytoid DC (pDC) and conventional myeloid DC (cDC) ${ }^{1-3}$ These $\mathrm{cDC}$ are further separated in $\mathrm{cDC} 1\left(\mathrm{CD} 141^{+}\right)$and $\mathrm{cDC} 2\left(\mathrm{CD} 1 \mathrm{c}^{+}\right) \mathrm{DC} .^{4-6}$ Initially, a fourth DC subset, slanDC, was identified based on the expression of M-DC8 (6-sulfo LacNAc or slan) and CD16. ${ }^{7-9}$ Recent studies by our own group and others, showed that these cells are actually more closely related to monocytes than to DC and they were renamed slan+ non-classical monocytes. ${ }^{10-12} \mathrm{DC}$ act as antigen presenting cells (APC) and orchestrate immune responses. Upon activation DC undergo a maturation process and up-regulate co-stimulatory molecules and secrete different types of cytokines, leading to antigen presentation and subsequent cellular immune responses. ${ }^{13}$ In several hematological malignancies immune dysregulation affecting the DC compartment has been reported, which
Haematologica 2022

Volume 107(3):655-667

\section{Correspondence:}

ARJAN A. VAN DE LOOSDRECHT

a.vandeloosdrecht@amsterdamumc.nl

Received: July 26, 2020.

Accepted: February 3, 2021.

Pre-published: February 11, 2021.

https://doi.org/10.3324/haematol.2020.268136

(C)2022 Ferrata Storti Foundation

Material published in Haematologica is covered by copyright. All rights are reserved to the Ferrata Storti Foundation. Use of published material is allowed under the following terms and conditions:

https://creativecommons.org/licenses/by-nc/4.0/legalcode. Copies of published material are allowed for personal or internal use. Sharing published material for non-commercial purposes is subject to the following conditions:

https://creativecommons.org/licenses/by-nc/4.0/legalcode, sect. 3. Reproducing and sharing published material for commercial purposes is not allowed without permission in writing from the publisher. 
might contribute to the pathogenesis of these malignancies and could be an important target for therapy. ${ }^{14-16}$ In MDS different types of immune cells are believed to play a role in pathophysiology. ${ }^{17,18}$ Low-risk disease is often characterized by an activated immune system in which pro-inflammatory cells are numerically increased. ${ }^{19,20}$ In contrast, it has been demonstrated in high-risk disease that immunosuppressive cell types, e.g., Tregs and myeloid derived suppressor cells (MDSC), are expanded and eventually facilitate immune escape and disease progression. ${ }^{20-23}$ Limited data is available on the role of DC in different MDS risk groups. Thus far, the focus of most studies has been on the frequencies and function of either in vitro generated monocyte-derived DC (MoDC) or total DC rather than functionally distinct DC subsets. ${ }^{24-27}$

In this study, we have investigated the frequencies of pDC and myeloid subsets (cDC and slan+ non-classical monocytes) in the BM and peripheral blood (PB) of different $\mathrm{MDS}$ risk group patients (i.e., low- and high risk based on the International Prognostic Scoring System [IPSS] and Revised IPSS [IPSS-R] or using the 2016 World Health Organization [WHO] classification) and compared them to normal BM (NBM) samples. Furthermore, we performed fluorescence in situ hybridization (FISH) analysis to demonstrate clonal involvement. A genome wide transcriptional analysis was carried out to find differences between healthy donor (HD) and MDS-derived subsets. In functional assays, their maturation and cytokine secreting capacity as well as their ability to induce $\mathrm{T}$-cell proliferation was assessed. Their reduced frequencies and a selective functional impairment, related to danger and tissue damage responsiveness, provide clues as to the role of these myeloid APC subsets in MDS progression.

\section{Methods}

\section{Patient and control samples}

In this study, $30 \mathrm{NBM}$ samples and $187 \mathrm{BM}$ and $26 \mathrm{~PB}$ samples of newly diagnosed MDS patients were used. Risk scores according to IPSS ${ }^{28}$ and the IPSS- ${ }^{29}$, were available for 150 and 136 patients, respectively. The 2016 WHO classification was available for 163 patients (details are given in the Online Supplementary Appendix and the Online Supplementary Table S1). NBM samples were obtained after written informed consent from patients who were undergoing cardiac surgery and were considered hematologically healthy (i.e., no cytopenia, normal morphology and normal flow cytometric profile). The study was approved by the local Institutional Review Board and was in accordance with the declaration of Helsinki.

\section{Enumeration of antigen presenting cells subsets and fluorescence in situ hybridization}

$\mathrm{PB}$ and $\mathrm{BM}$ cells were analyzed on a flow cytometer (FACSCanto $^{\text {TM }}$, BD Biosciences) after incubation with a panel of monoclonal antibodies (see the Online Supplementary Appendix for details). After debris and doublet exclusion, cell subtypes were identified in the $\mathrm{CD} 45^{+}$compartment. CD141, CD1c, CD303 and M-DC8/CD16 were used for the identification of cDC1, cDC2, pDC and slan+ monocytes, respectively (Figure $1 \mathrm{~A})$. Frequencies of all populations were calculated as percentages of CD45+ mononuclear cells.

Three MDS samples with a known cytogenetic aberrancy were used for the isolation of $\mathrm{cDC} 2$ and slan+ monocytes and subsequent FISH analysis (details are given in the Online Supplementary Appendix).

\section{Functional assays and multidimensional mass cytometry}

The maturation capacity, the secretion of cytokines and the ability to induce T-cell proliferation was tested for MDS BM- and NBM-derived $\mathrm{cDC} 2$ and slan+ non-classical monocytes. See the Online Supplementary Table S1 for clinical data. A multi-parameter deep-phenotyping strategy, known as cytometry by time-of-flight (CyTOF), was used for T cells cultured in the presence of MDSderived or healthy PB-derived slan+ monocytes. DC subsets could not be included in this experiment because of low cell numbers. See the Online Supplementary Appendix file for technical details.

\section{Microarray transcriptional analysis}

RNA was isolated from MDS BM- and NBM-derived cDC2 and slan+ monocytes (5.000-67.000 cells) and amplified using the Ovation Pico WTA System V2 (NuGen, San Carlos, CA) as previously described.11 RNA was labeled with the Encore Biotin Module Kit (NuGEN) and $5 \mathrm{mg}$ of cDNA from each sample was hybridized to Human Transcriptome Arrays 2.0 microarrays (Affymetrix) and signals were scanned by Affymetrix GeneChip Scanner 3000 7G. See the Online Supplementary Appendix for details on data analysis. The microarray data have been deposited in the GEO public database under the accession number: GSE161058.

\section{Statistical analysis}

Graphpad Prism 6 software (San Diego, USA) was used for flow cytometry and functional data analysis and graphic display. For two-group comparisons, differences were assessed by applying a non-parametric Mann-Whitney U test. Multi-group comparisons were analyzed with a Kruskal-Wallis with Dunn's multiple comparisons test. The non-parametric Spearman's correlation test was used for correlations. A $P$-value of $<0.05$ was considered significant.

\section{Results}

\section{Dendritic cell frequencies are reduced in the bone marrow of MDS patients}

Frequencies of different DC subsets and slan+ monocytes were analyzed in BM samples of 187 newly diagnosed myelodysplastic syndrome patients (detailed in Table 1) and compared to 30 NBM samples. An eightcolour flow cytometry panel was used for the detection of $\mathrm{CD}_{0} 3^{+} \mathrm{pDC}, \mathrm{CD} 141^{\mathrm{hi}} \mathrm{cDC} 1, \mathrm{CD} 1 \mathrm{c}^{+} \mathrm{cDC} 2$ and $\mathrm{M}-\mathrm{DC} 8^{+} / \mathrm{CD} 6^{+}$non-classical monocytes (Figure 1A). Except for pDC, all subsets showed significantly lower frequencies in MDS-derived BM compared to NBM (Figure $1 \mathrm{~B}$; NBM vs. MDS BM: cDC1 $0.048 \%$ vs. $0.030 \%$, cDC2 $0.67 \%$ vs. $0.54 \%$ and slan $+0.36 \%$ vs. $0.24 \%$ ). pDC rates were increased in MDS BM (NBM, 0.76\%; MDS BM, $0.91 \%)$. This was mainly observed in cases that were associated with low blast counts (Figure 1C; NBM, 0.76\%; (RS-)SLD/MLD, 1.11\%; EB-1/EB-2, 0.63\%). For CDC1 and CDC2, frequencies gradually decreased in classification groups associated with higher risk MDS (Figure 1C; NBM, $0.048 \%$; (RS-)SLD/MLD, $0.038 \%$; EB-1/EB-2, $0.015 \%$ and NBM, 0.67\%; (RS-)SLD/MLD, 0.59\%; EB-1/EB-2, 0.44\%, respectively). Also, for slan+ monocytes lowest frequencies were found in the EB-1/EB-2 classification group (NBM，0.36\%; (RS-)SLD/MLD，0.24\%; EB-1/EB-2, 
$0.23 \%$ ). Using the IPSS and IPSS-R risk stratification, similar results were observed. Patients within higher risk groups showed lower frequencies of cDC (Figure 1D and E). Furthermore, $\mathrm{MDS}$-derived paired $\mathrm{BM}$ and $\mathrm{PB}$ samples showed strong correlations for frequencies of designated subsets, except for cDC1 (Figure 1F).

In order to assess clonal involvement of isolated cDC2 and slan+ non-classical monocytes (due to limited cell availability $\mathrm{cDC1}$ was not tested), three different MDS samples with a known cytogenetic aberration (del5q, trisomy 8 and monosomy 7) were selected for FISH analysis. In all tested cases, cDC2 were highly involved in the dysplastic clone. Due to the limited cell numbers of slan+ monocytes, a clear conclusion could only be drawn for the del $5 q$ analysis. In this patient slan+ monocytes were also highly involved in the dysplastic clone. As expected, CD34 ${ }^{+}$progenitor cells were also clonally involved whereas B cells were not (Figure 2).

\section{Immunological gene sets related to danger response are under-represented in myelodysplastic syndrome-derived antigen presenting cells}

In order to screen for functional differences between $\mathrm{HD}$ - and MDS-derived $\mathrm{cDC}$ and slan+ monocytes a genome-wide transcriptional profiling study was performed. cDC2 and slan+ monocytes were sorted from previously stored $\mathrm{HD}(\mathrm{n}=3)$ and $\mathrm{MDS}(\mathrm{n}=4)$ BM samples. Because of low cell numbers, cDC1 were not included in this study. Patient samples were selected based on their 2016 WHO classification group in order to create a more homogenous set of samples. All patients were diagnosed with RS-MLD, were in very low to intermediate IPSS-R risk categories and, apart from one patient who had a 45,X,-Y [3] / 46,XY [7] karyotype, showed normal cytogenetics. In total, 135.750 genes were found to be expressed (including non-coding genes). Using ANOVA testing the number of differentially expressing genes (DEG) for coding transcripts between $\mathrm{HD}$ and MDS was 1.922 for cDC2 and 2.415 for slan+ monocytes. In cDC2 1.075 genes were under-expressed in MDS compared to $\mathrm{HD}$ and 847 genes were over-expressed. Slan+ monocytes showed 1.655 under-expressed and 760 overexpressed genes. Hierarchical clustering showed a clear separation between $\mathrm{HD}$ - and MDS-derived samples for both subsets (Figure 3A). Volcano plots were used to show over- and under-expressed genes in $\mathrm{HD}$ compared to MDS with fold change levels of $<-2.5$ or $>2.5$ and a gene level $P$-value $<0.05$ (Figure $3 \mathrm{~B})$. Next, DEGs were used for further pathway analysis in order to find biologically relevant differences between $\mathrm{HD}$ and MDS subsets. Transcripts that were under-expressed in MDS were imported in the STRING v10.5 database and a gene ontology (GO) term enrichment analysis was performed. Six of the most enriched pathways are shown for both subsets (Figure 3C). Pathways highly involved in proinflammatory processes and innate immune activation were under-represented in MDS as compared to $\mathrm{HD}$. When uploading lists containing over-expressed genes in MDS, hardly any immune response-related pathways were enriched (not shown). Interestingly, for cDC2 pathways related to apoptosis were enriched in biological processes as well as in KEGG pathways. For slan+ monocytes this analysis mainly yielded pathways containing metabolic- and general cell biology-related processes. In order to confirm these analyses gene set enrichment analysis (GSEA) was executed. Twenty gene sets involved in immunological processes were selected from the Broad Institute database and tested for enrichment in either $\mathrm{HD}$ samples or MDS samples. For cDC2 5 gene sets were significantly enriched in $\mathrm{HD}$ (with a nominal $P$ value $<0.01$ ) and none of them was enriched in MDS. Slan+ monocytes showed five significantly enriched gene sets in $\mathrm{HD}$ and two in MDS (Figure $3 \mathrm{D}$ and $\mathrm{E}$ ). For each cell subset an example enrichment plot is shown. Genes that contribute to the core enrichment for this specified gene set are displayed in a heatmap (67 genes for $\mathrm{cDC} 2,75$ of 280 are shown for slan+ monocytes). For cDC2, a great part of these genes consisted of pattern recognition receptors, such as the toll-like receptors (TLR $1,2,3,5,7,8$ and 10), C-type lectin receptors (CLEC4A, $E, C$ and $C L E C 6 A)$ and $C D 180$. For slan+ monocytes also Fc- $\gamma$ receptors (FCGR1A, FCGR2A/B and FCGR3A) and

Table 1. Patient and control characteristics

\begin{tabular}{lcc} 
Number & Bone marrow & Peripheral blood \\
HD & 217 & 26 \\
MDS & 30 & - \\
Age - mean, y & 187 & 26 \\
HD & & \\
MDS & 62 & - \\
\hline
\end{tabular}

Sex

$\begin{array}{lcc}\text { HD - male/female } & 20 / 10 & - \\ \text { MDS - male/female } & 134 / 53 & 17 / 9 \\ \text { IPSS } & & \\ \text { Low risk } & 53 & 10 \\ \text { Intermediate-1 } & 71 & 10 \\ \text { Intermediate-2 } & 21 & - \\ \text { High risk } & 5 & - \\ \text { Missing IPSS score } & 37 & 6\end{array}$

IPSS-R

Very low risk

Low risk

Intermediate risk

High risk

Very high risk

Missing IPSS-R score

$29 \quad 5$

WHO

\begin{tabular}{lcc} 
MDS-SLD & 11 & 5 \\
MDS-MLD & 65 & 4 \\
MDS-RS-SLD & 8 & 2 \\
MDS-RS-MLD & 31 & 7 \\
MDS-EB-1 & 24 & 3 \\
MDS-EB-2 & 24 & - \\
Missing WHO 2016 classification & 24 & 5 \\
\% Blasts & & \\
$<5 \%$ & 116 & 13 \\
$\geq 5 \%$ & 51 & 5 \\
\hline
\end{tabular}

MDS: myelodysplastic syndromes; EB: excess blasts; HD: healthy donor; IPSS(-R): (Revised) International Prognostic Scoring System; MLD: multilineage dysplasia; RS: ring sideroblasts; SLD: single lineage dysplasia; WHO: World Health Organisation; y: years. 
A
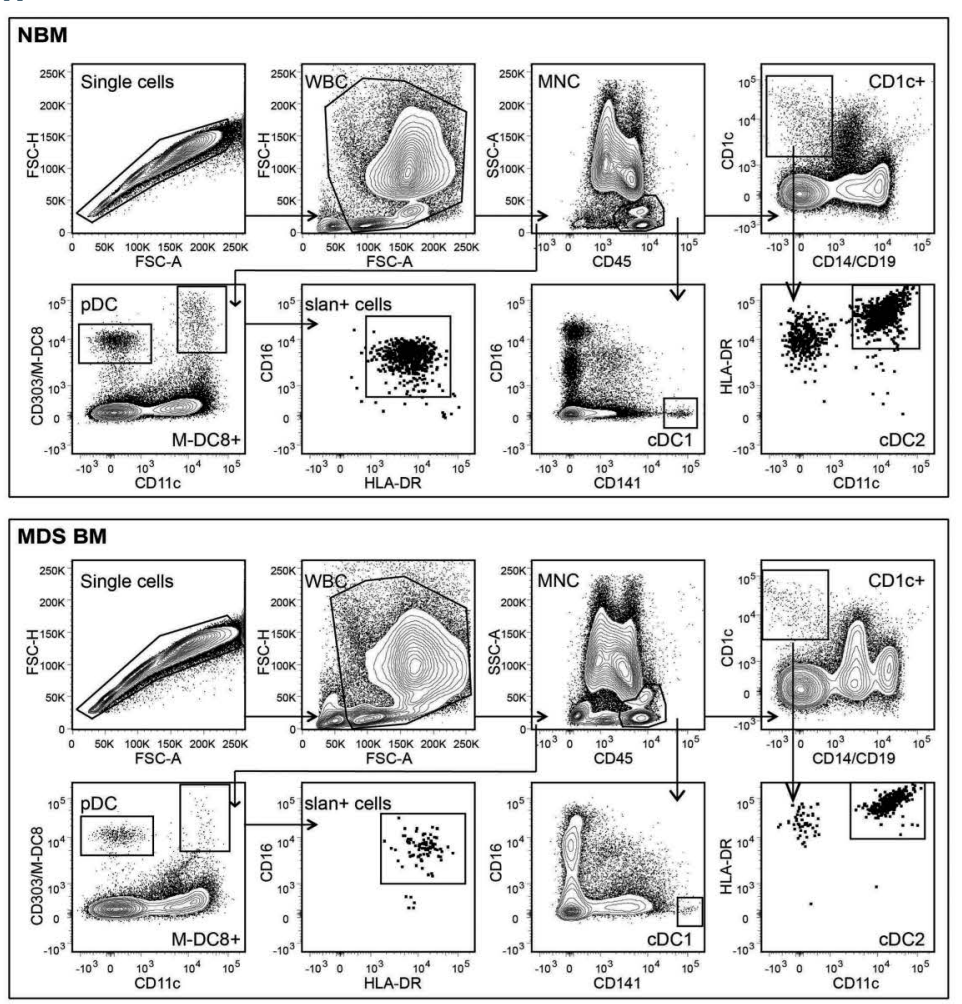

B

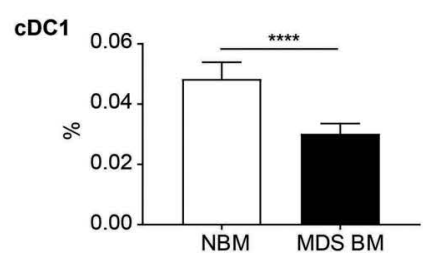

CDC2

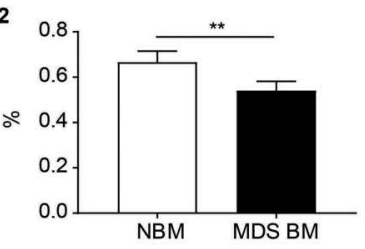

Slan+

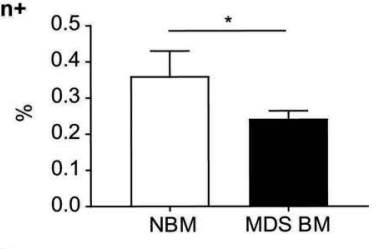

pDC

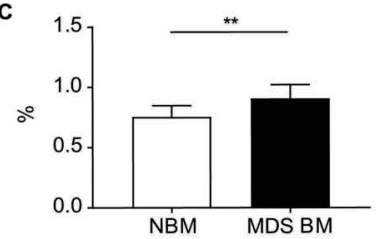

C
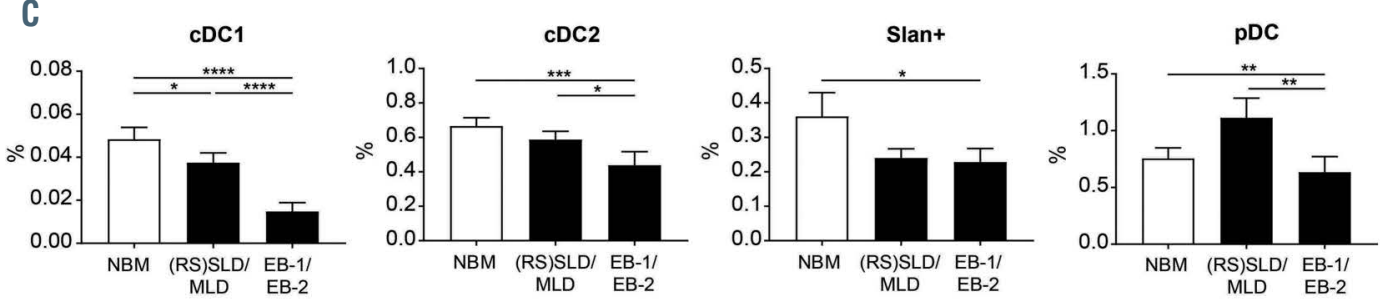

D
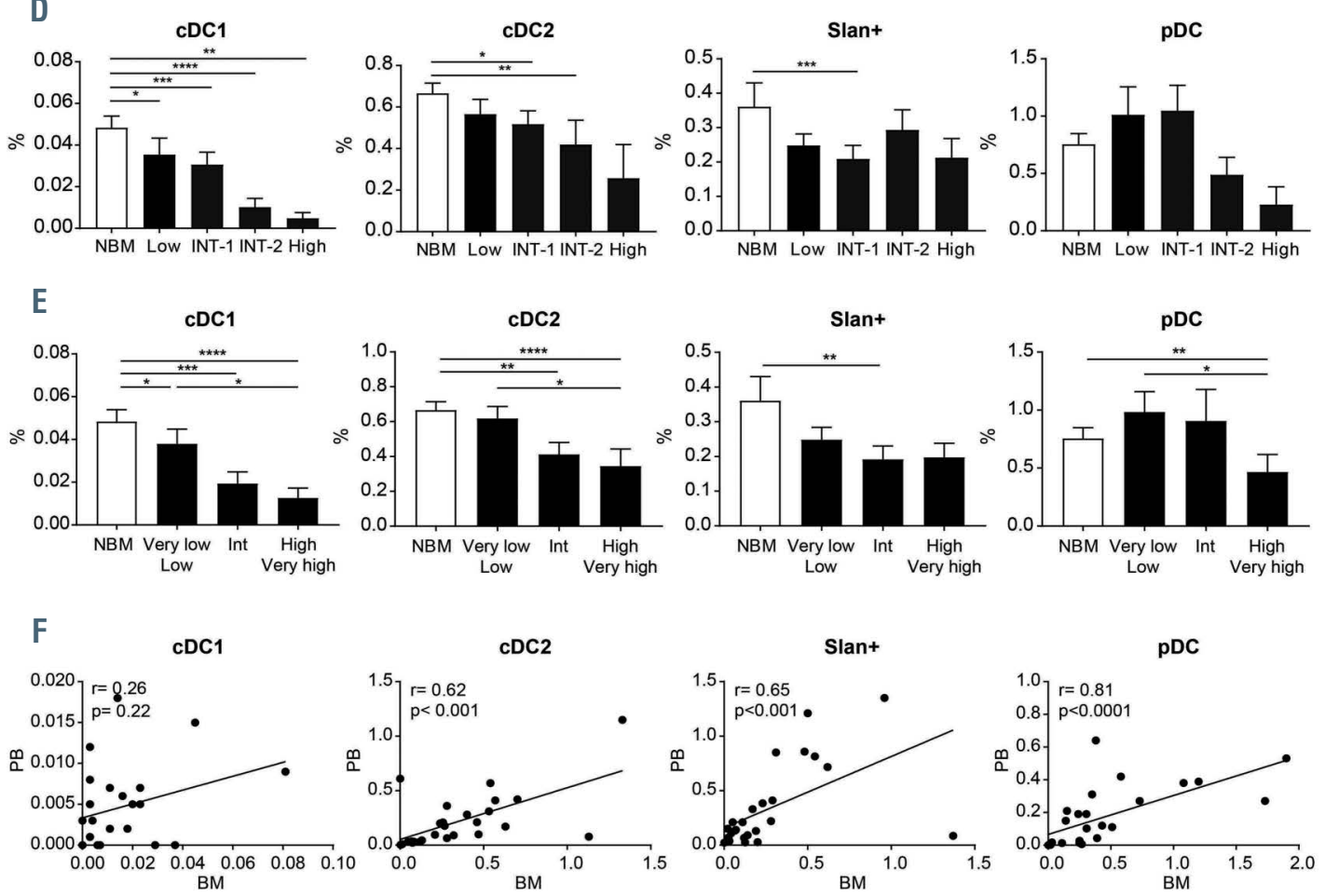

Figure 1. Legend on following page. 
Figure 1. Cell subset enumeration in myelodysplastic syndrome- and healthy-derived bone marrow and peripheral blood. (A) Gating strategy of dendritic cells (DC) and slan+ non-classical monocytes in normal bone marrow (NBM) and myelodysplastic syndromes (MDS)-derived BM. After debris and doublet exclusion, $\mathrm{CD} 45^{+}$mononuclear cells were gated. Then plasmacytoid DC ( $\left.\mathrm{pDC}\right)$, myeloid $\mathrm{DC}(\mathrm{CDC} 1$ and $\mathrm{cDC} 2)$ and slan+ monocytes were identified based on the expression of CD141 ${ }^{\text {high }}$, CD1C and M-DC8/CD16, respectively. (B) Frequencies of different cell subsets in normal bone marrow (NBM) compared to MDS BM. In total 30 NBM samples and 187 MDS BM samples were used. Percentages were calculated from the mononuclear cell fraction. Mean frequencies \pm standard error of the mean $(\mathrm{SEM})$ are given (NBM vs. MDS BM: pDC $0.76 \%$ SEM \pm 0.09 vs. $0.91 \%$ SEM \pm 0.11 , cDC1 $0.048 \% \mathrm{SEM} \pm 0.006$ vs. $0.030 \% \mathrm{SEM} \pm 0.003, \mathrm{cDC} 20.67 \% \mathrm{SEM} \pm$ 0.05 vs. $0.54 \%$ SEM \pm 0.04 and slan $+0.36 \%$ SEM \pm 0.07 vs. $0.24 \%$ SEM \pm 0.02 ). (C) Cell frequencies in different classification groups according to the 2016 World Health Organization (WHO) classification. Patients having a higher blast count-related 2016 WHO classification (EB-1/EB-2) show lower percentages of DC and slan+ monocytes compared to NBM and lower risk groups (SLD/MLD/RS-SLD/RS-MLD). NBM ( $n=30)$ vs. (RS-)SLD/MLD ( $n=115)$ vs. EB-1/EB-2 ( $=48$ ): $p D C$ $0.76 \%$ vs. $1.11 \%$ vs. $0.63 \%$, cDC1 $0.048 \%$ vs. $0.038 \%$ vs. $0.015 \%$, cDC2 $0.67 \%$ vs. $0.59 \%$ vs. $0.44 \%$, slan+ $0.36 \%$ vs. $0.24 \%$ vs. $0.23 \%$. (D) Cell frequencies in different risk groups within the International Prognostic Scoring System (IPSS). The percentages of myeloid DC subsets decrease gradually in higher risk groups. NBM $(n=30)$ vs. low risk $(n=49)$ vs. intermediate- $1(n=71)$ vs. intermediate- $2(n=21)$ vs. high risk $(n=5): c D C 10.048 \%$ vs. $0.035 \%$ vs. $0.031 \%$ vs. $0.010 \%$ vs. $0.005 \%$, cDC2 $0.67 \%$ vs. $0.57 \%$ vs. $0.52 \%$ vs. $0.42 \%$ vs. $0.26 \%$. (E) Cell frequencies in different risk groups within the IPSS-R. Again, differences between subgroups are mainly seen in DC subsets. Higher risk groups show lower percentages of DC compared to NBM and lower risk groups. NBM ( $n=30$ ) vs. very low/low risk $(n=77)$ vs. intermediate risk $(n=32)$ vs. high/very high risk $(n=27)$ : $c D C 10.048 \%$ vs. $0.038 \%$ vs. $0.019 \%$ vs. $0.013 \%$, $c D C 20.67 \%$ vs. $0.62 \%$ vs. $0.41 \%$ vs. $0.35 \%$. (F) Correlation of cell frequencies in MDS-derived peripheral blood (PB) and BM samples. In total, 26 paired MDS samples were included. The non-parametric Spearman's correlation test was used to find significant correlations between frequencies in $\mathrm{PB}$ and $\mathrm{BM}$. $* P<0.05$, $* * P<0.01$, $* \star \star P<0.001$, $\star * * * P<0.0001$. EB: excess blasts; MLD: multilineage dysplasia; RS-MLD: ring sideroblasts with multilineage dysplasia; RS-SLD: ring sideroblasts with single lineage dysplasia; SLD: single lineage dysplasia.

$\mathrm{CDC} 2$
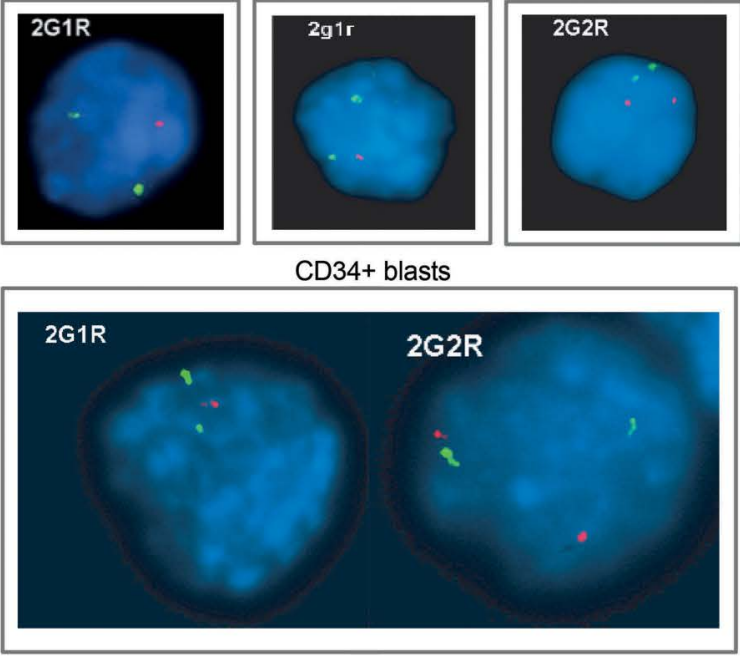

Slan+

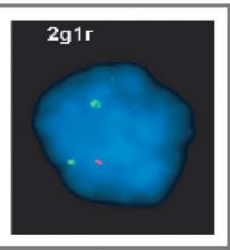

CD34+ blasts

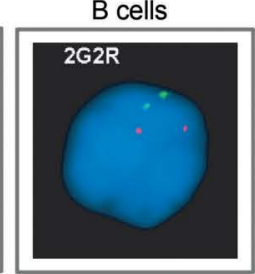

.
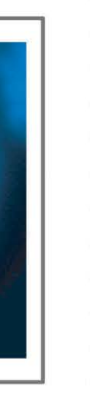

\begin{tabular}{l|c|c}
\hline Cell type & \multicolumn{2}{|c}{ FISH results: number of cells } \\
\hline & Monosomy 7 & Two chromosome 7 \\
\hline Whole bone marrow & 57 & 43 \\
\hline cDC2 & 92 & 8 \\
\hline Slan+ & 2 & 20 \\
\hline B cells & 0 & 100 \\
\hline CD34+ blasts & 96 & 4 \\
\hline & Del 5q & Normal chromosome 5 \\
\hline Whole bone marrow & 163 & 37 \\
\hline cDC2 & 20 & 1 \\
\hline Slan+ & 95 & 98 \\
\hline B cells & 2 & 4 \\
\hline CD34+ blasts & 96 & 44 \\
\hline & Trisomy 8 & 39 \\
\hline Whole bone marrow & 56 & 6 \\
\hline cDC2 & 61 & 91 \\
\hline Slan+ & 10 & 23 \\
\hline B cells & 0 & Two chromosome 8 \\
\hline CD34+ blasts & 77 & \\
\hline & & \\
\hline
\end{tabular}

Figure 2. Clonal involvement of dendritic cells subsets and slan+ monocytes. Fluorescence in situ hybridization (FISH) analysis of sorted cells, including B cells and $\mathrm{CD}_{4} 4^{+}$blast cells, with a known cytogenetic aberrancy. In three tested cases (monosomy 7 , del $5 \mathrm{q}$ and trisomy 8 ), isolated cDC2 and CD34 ${ }^{+}$blast cells were highly involved in the dysplastic clone, whereas B cells were not involved. Slan+ monocytes showed clonal involvement in del5q. Interphase FISH on whole bone marrow samples showed both an aberrant and a normal cell line. A representative FISH analysis is shown in which interphase cells are hybridized with the chromosome $5 q$ probe displayed in red and 5p probe displayed in green (LSI EGR1(5q31)/D5S23,D5S721(5p15.2) Dual Colour Probe Set). Loss of 5q is seen in CD34 ${ }^{+}$blasts, cDC2 and slan+ monocytes (2G1R), but not in B cells (2G2R).

complement receptors $(C 3 A R 1)$ are at the top of this list. Further leading-edge analysis using all five gene sets was performed to identify the genes that highly account for the gene set's enrichment signal (Figure 4A). There was a great overlap of genes that formed the leading-edge subset between all gene sets (Figure 4B). In total, 418 genes were found to form the leading-edge subset for $\mathrm{CDC} 2$ of which 32 were present in all five gene sets. For slan+ monocytes 353 genes formed the leading-edge subset. Of them, 39 genes were found in all five gene sets. These genes were considered most relevant because they form the core of the enrichment (Figure 4C). Again, both lists with lead targets consisted of multiple pattern recognition receptors, which suggests an overall diminished capacity for sensing pathogen/damage associated molecular patterns (PAMP/DAMP) by MDS-derived cells. This was further confirmed by the fact that also genes that were highly involved in subsequent down-stream cell signaling, such as BTK, CARD9, IRAK4,IRF3/7, MyD88, $S Y K$, and usually lead to activation of pro-inflammatory processes, were down-regulated in MDS-derived APC.

\section{Myelodysplastic syndrome-derived cells show reduced T-cell priming capacities and clear Th1/2-type T cell skewing}

Next, in order to confirm the hypothesis that was formed from the gene expression profiling data, functional capacities of $\mathrm{cDC} 2$ and slan+ monocytes were tested. Upon stimulation with LPS and R848, a combination of proven synergistically working TLR ligands, ${ }^{33} \mathrm{cDC} 2$ showed upregulation of co-stimulatory molecules. In contrast, slan+ monocytes were unable to upregulate maturation markers (Figure 5A). Again, cDC1 were not tested because of low frequencies. There was no statistical difference in maturation capacity for $\mathrm{cDC} 2$ when they were compared to NBM-derived cDC2. Slan+ monocytes showed a significantly reduced ability to upregulate CD80 upon stimulation compared to their equivalents in NBM (Figure 5B). In order to investigate their cytokine secreting capacity, MDS-derived cDC2 and slan+ monocytes were isolated and either left non-stimulated or stimulated overnight with TLR ligands. Culture supernatants were tested for the presence of different cytokines. Compared 
A
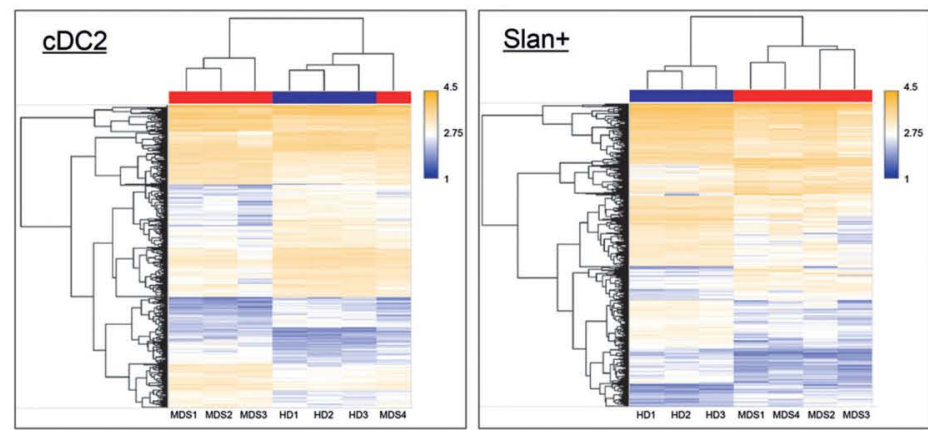

B
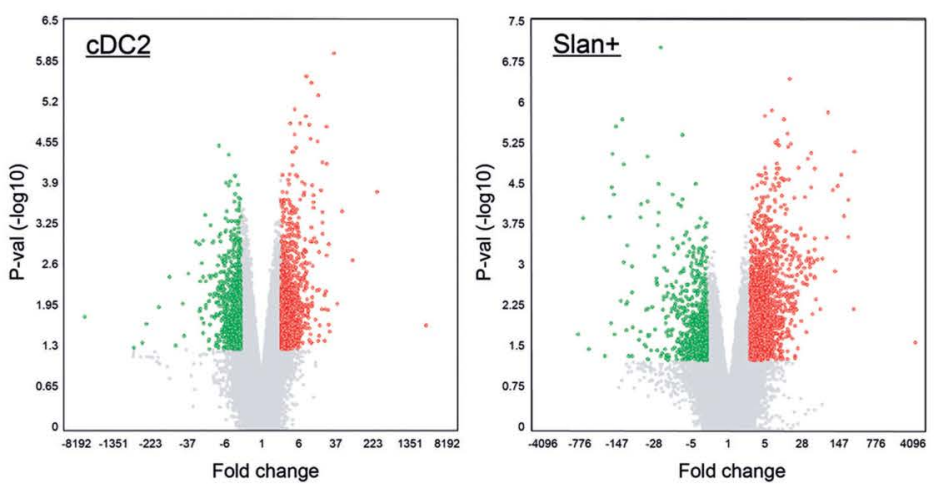

Figure 3. Transcriptomic comparison between healthy donor- and myelodysplastic syndrome-derived cell subsets. Three healthy donor (HD)-derived samples and four myelodysplastic syndromes (MDS)-derived samples were used for the isolation of $\mathrm{CDC} 2$ and slan+ monocytes and subsequent microarray analysis. (A) Hierarchical clustering, based on differentially expressed genes, of replicate samples for cDC2 and slan+ monocytes. Heatmap visualization is used to show transcript clustering for the two different conditions (HD vs. MDS). (B) Volcano plots showing overand under-expressed genes in red and green, respectively, in HD compared to MDS. A cut-off of -2.5 / 2.5 for foldchange and a $P$-value $<0.05$ were used to show results. (C) Pathway analyses for transcripts that are under-expressed in MDS compared to HD for CDC2 and slan+ monocytes. Coding differentially expressed genes ( 774 genes for $\mathrm{CDC} 2$ and 987 genes for slan+ monocytes) were selected and imported into the STRING v10.5 bioinformatics tool. Six enriched biological processes with lowest false discovery rate (FDR) are shown for CDC2 and slan+ monocytes (in total, eight enriched pathways were found for CDC2 and 383 for slan+ monocytes). (D and E) Gene set enrichment analysis for HD- and MDS-derived CDC2 (D) and slan+ monocytes $(\mathrm{E})$. For both subsets five gene sets were enriched in HD. An enrichment plot is displayed for each subset. Heatmaps show the core enriched genes (67 for cDC2 and the top 75 of 280 for slan+ monocytes) with interesting genes highlighted by black stars. GO: gene ontology; NES: normalized enrichment score; Nom P-value, nominal $P$-value.
C GO term enrichment analysis - Biological processes

\begin{tabular}{lll}
\hline Pathway ID & Pathway description & FDR \\
\hline cDC2 & & \\
GO.0008152 & metabolic process & 0.0019 \\
GO.0044237 & cellular metabolic process & 0.0020 \\
GO.0044710 & single-organism metabolic process & 0.0020 \\
GO.0045087 & innate immune response & 0.0020 \\
GO.0006996 & organelle organization & 0.0031 \\
GO.0044283 & small molecule biosynthetic process & 0.0187 \\
\hline
\end{tabular}

\section{GO - Biological processes - CDC2}

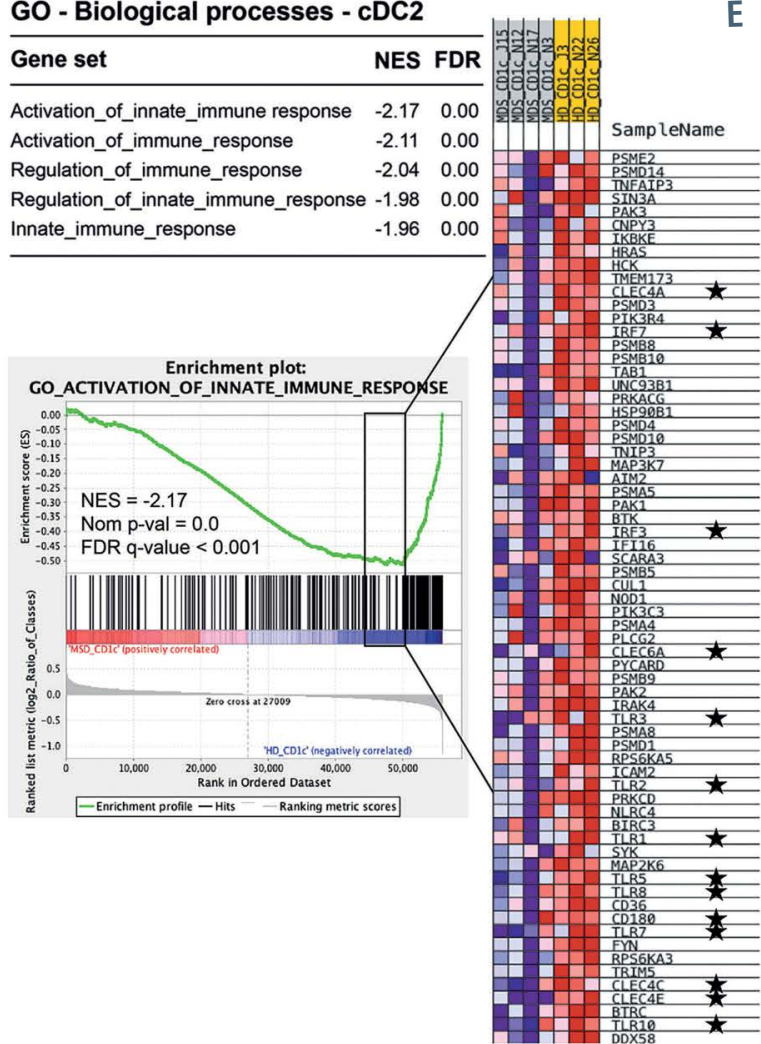

GO term enrichment analysis - Biological processes

\begin{tabular}{lll}
\hline Pathway ID & Pathway description & FDR \\
\hline Slan+ & & \\
GO.0006955 & immune response & 0.0000 \\
GO.0002252 & immune effector response & 0.0000 \\
GO.0002682 & regulation of immune system process & 0.0000 \\
GO.0002376 & immune system process & 0.0000 \\
GO.0048583 & regulation of response to stimulus & 0.0000 \\
GO.0050776 & regulation of immune response & 0.0000 \\
\hline
\end{tabular}

E GO - Biological processes - Slan+

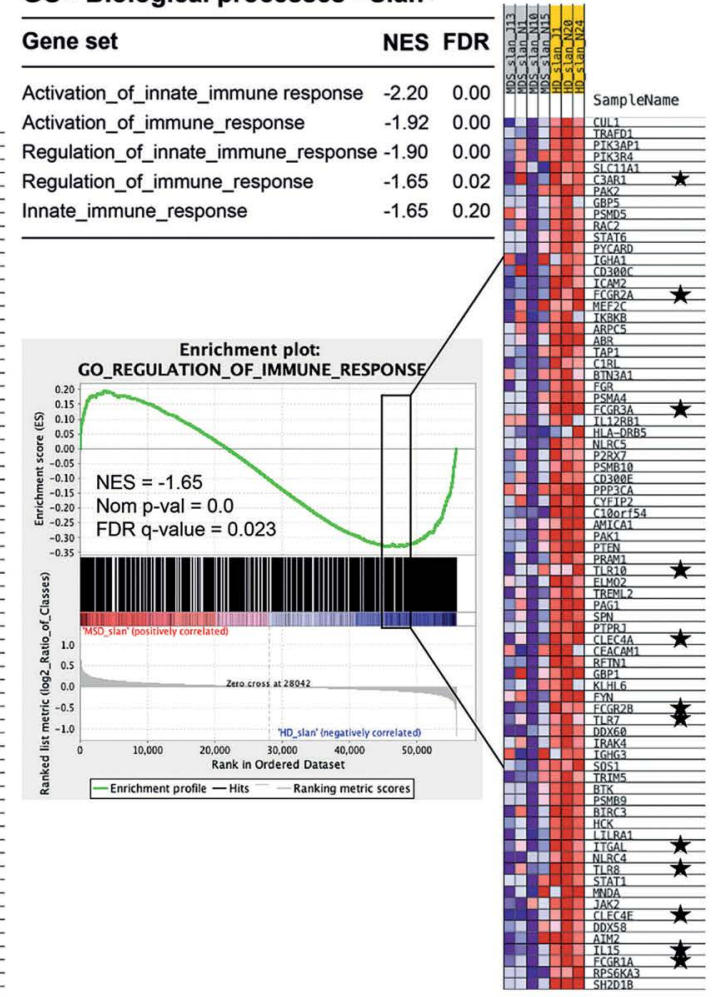


A

\begin{tabular}{lccc} 
A & $\begin{array}{l}\text { \# } \\
\text { members }\end{array}$ & $\begin{array}{l}\text { \# in } \\
\text { signal }\end{array}$ & \% \\
\hline Activation_of_innate_immune response & 200 & 67 & $34 \%$ \\
Activation_of_immune_response & 407 & 127 & $31 \%$ \\
Regulation_of_immune_response & 819 & 291 & $36 \%$ \\
Regulation_of_innate_immune_response & 352 & 121 & $34 \%$ \\
Innate_immune_response & 586 & 234 & $40 \%$ \\
\hline
\end{tabular}

\begin{tabular}{lccc} 
Slan+ & $\begin{array}{l}\text { \# } \\
\text { members }\end{array}$ & $\begin{array}{l}\text { \# in } \\
\text { signal }\end{array}$ & $\%$ \\
\hline Activation_of_innate_immune response & 200 & 82 & $41 \%$ \\
Activation_of_immune_response & 407 & 140 & $34 \%$ \\
Regulation_of_innate_immune_response & 352 & 130 & $37 \%$ \\
Regulation_of_immune_response & 819 & 280 & $34 \%$ \\
Innate_immune_response & 586 & 155 & $26 \%$ \\
\hline
\end{tabular}

B

cDC2
1. 2. 3. 4. 5 .
1. Regulation of immune response
2. Activation_of_immune_response
3. Activation_of_innate_immune_respons
4. Regulation_of_innate_immune_response
5. Innate immune response

C

cDC2

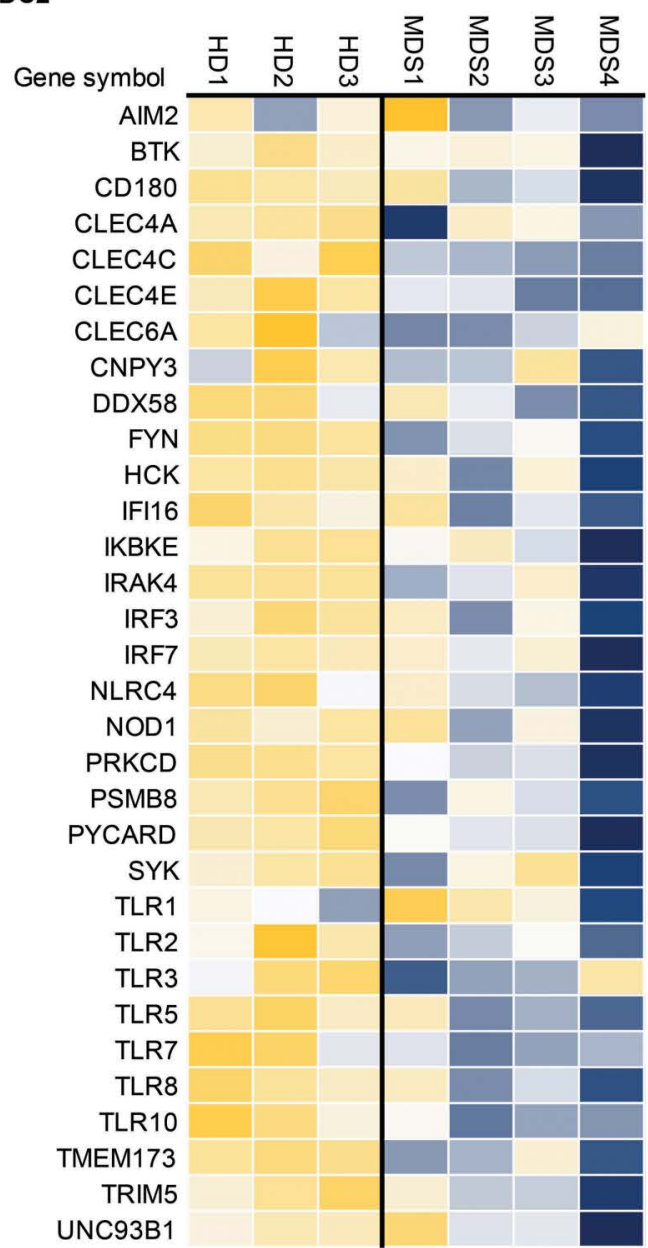

Low High

Expression
Slan+

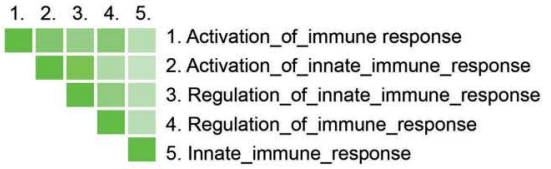

Slan+

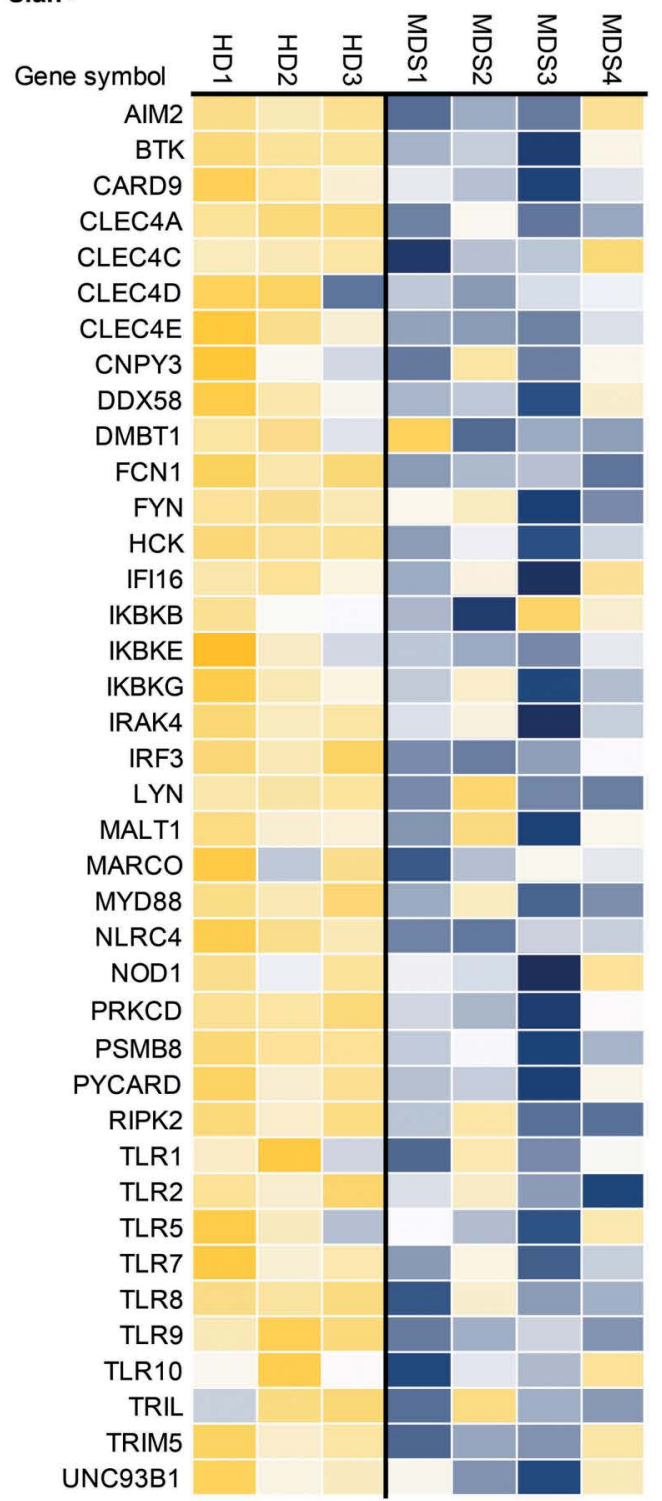

Figure 4. Leading-Edge Analysis. (A) Leading-Edge Analysis using five gene sets for both cell subsets. The tables show total number of genes that are present in a specified gene set and the number and percentage of genes that were considered to form the leading-edge subset of that gene set. (B) A set-to-set analysis for cDC2 and slan+ monocytes. Overlap in leading-edge genes between gene sets are displayed using a color intensity graph. A dark green cell indicates that sets have the same leading-edge genes. (C) Heatmap of the leading-edge subset for CDC2 and slan+ monocytes. Genes displayed are present in the leading-edge subset of all five gene sets. The heatmaps show relative expression levels per gene between healthy donor (HD) and myelodysplastic syndrome (MDS) samples. FDR: false discovery rate; GO: gene ontology; NES: normalized enrichment score; Nom $P$-value: nominal $P$-value. 
A
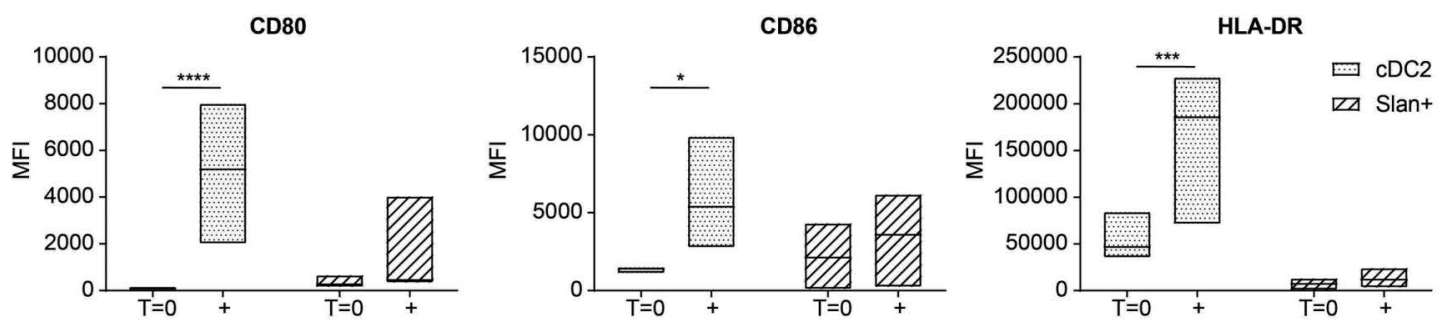

B


ロ NBM ロ MDSBM

C
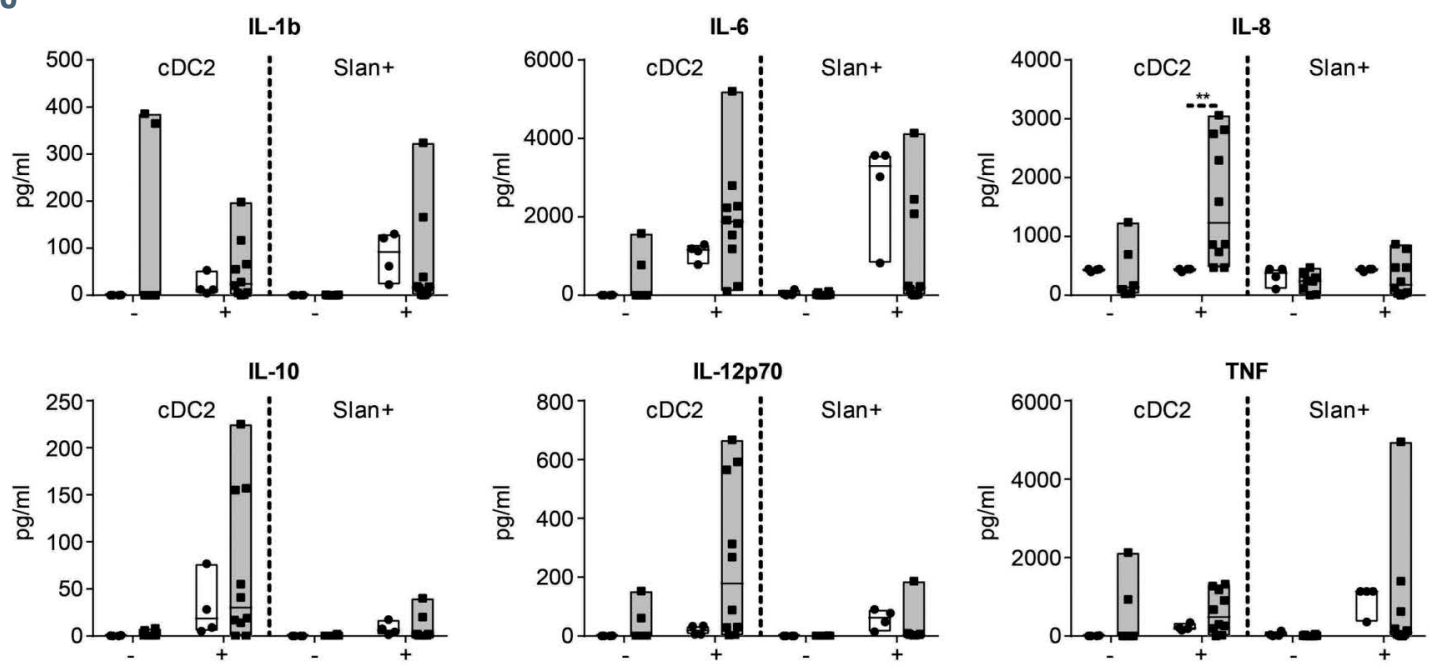

口NBM $\square$ MDSBM
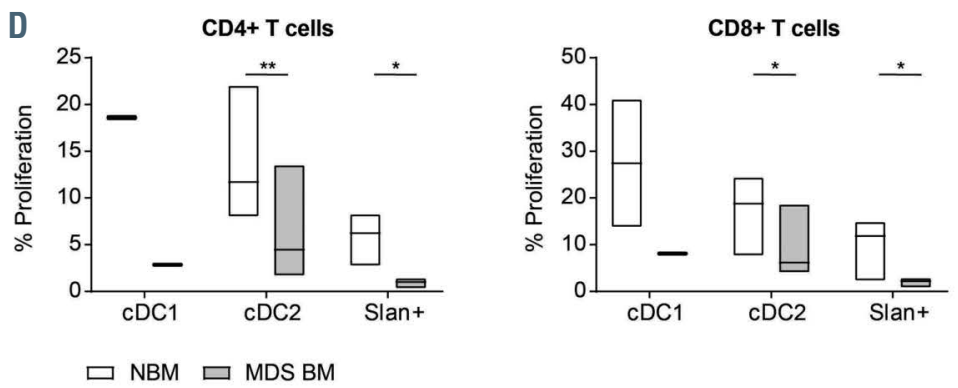

Figure 5. Functional capacities of dendritic cell subsets and slan+ monocytes. (A) Maturation capacity of CDC2 and slan+ monocytes in myelodysplastic syndrome (MDS) bone marrow (BM) upon toll-like receptor (TLR)-stimulation. Expression levels of CD80, CD86 and HLA-DR were assessed by flow cytometry at baseline ( $T=0$ ) and after overnight stimulation with LPS + R848 (+). Mean fluorescence intensity (MFI) values of these three markers were measured. Median values of 4-7 experiments are shown. (B) Up-regulation of CD80, CD86 and HLA-DR after overnight TLR-stimulation in normal bone marrow (NBM)- $(n=4)$ and MDS-derived ( $n=4-7)$ cDC2

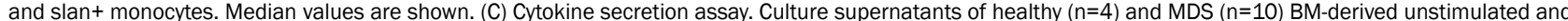
stimulated CDC2 and slan+ monocytes were analyzed for the presence of different cytokines by cytometric bead array. Median values are shown. (D) Allogeneic mixed leukocyte reaction (MLR). Peripheral blood lymphocytes (PBL) were labeled with carboxyfluorescein succinimidyl ester (CFSE) and co-cultured with healthy ( $n=2-4)$ or MDS-derived ( $n=1-5$ ) stimulated CDC1, $C D C 2$ or slan+ monocytes. The percentage of CFSE-diluted T cells was determined by flow cytometry. Median values of different experiments are shown. $* P<0.05, * * P<0.01, * * * P<0.001, * * * * P<0.0001$. pg: picogram. 


\section{A Healthy donor}

IFN-y

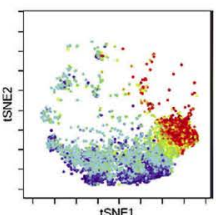

Day 5

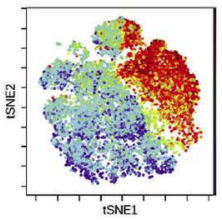

IFN-y

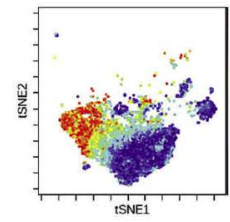

Day 5

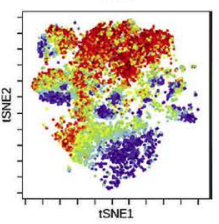

IL-4


IL-4
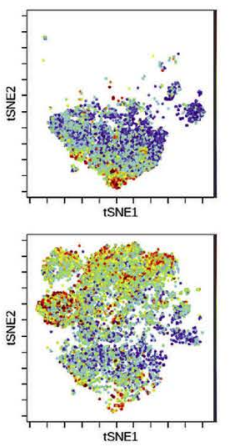

IL-17
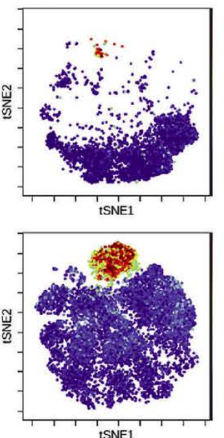

IL-17
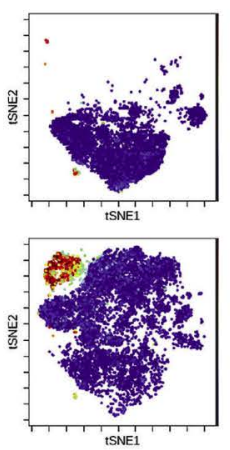

IL-10
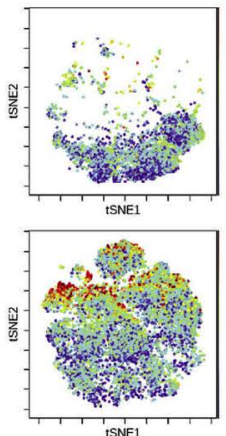

IL-10
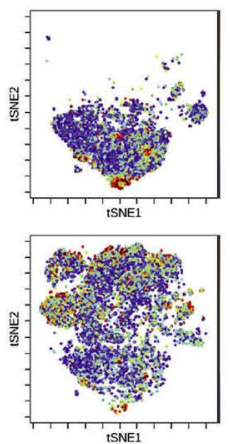

FoxP3
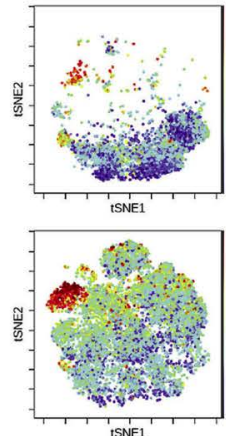

FoxP3
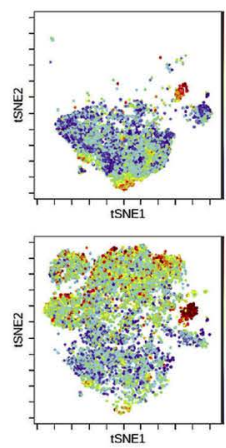

\section{B Healthy donor}

Day 0

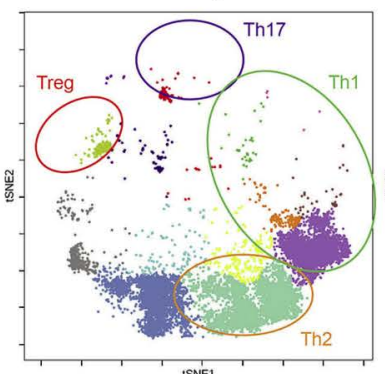

MDS

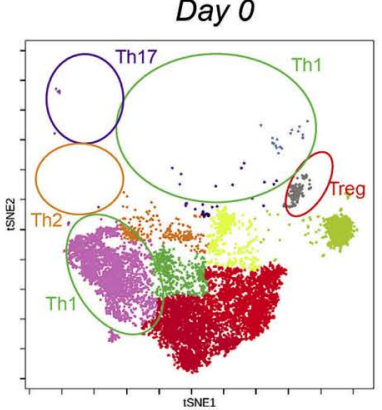

Day 5

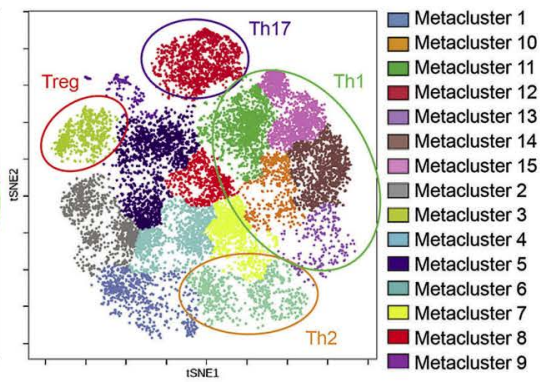

Day 5

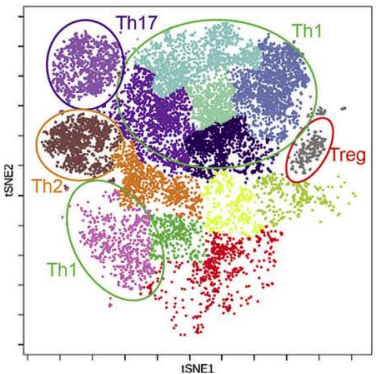

$\square$ Metacluster 1 $\square$ Metacluster 10 $\square$ Metacluster 11 $\square$ Metacluster 12 $\square$ Metacluster 13 $\square$ Metacluster 14 $\square$ Metacluster 2 $\square$ Metacluster 3 $\square$ Metacluster 4 - Metacluster 5 $\square$ Metacluster 6 $\square$ Metacluster 7 $\square$ Metacluster 8 Metacluster 9

\begin{tabular}{c|c|c|c}
\hline T-cell subset & Day 0 & Day 5 & Difference \\
\hline Th1 & $31.4 \%$ & $41.0 \%$ & $-9.6 \%$ \\
\hline Th2 & $33.6 \%$ & $2.7 \%$ & $-30.9 \%$ \\
\hline Th17 & $1.7 \%$ & $11.7 \%$ & $-10.0 \%$ \\
\hline Treg & $1.7 \%$ & $4.3 \%$ & $-2.6 \%$ \\
\hline
\end{tabular}

Figure 6. Mass cytometry of T cells co-cultured in the presence of healty donor- or myelodysplastic syndrome-derived slan+ monocytes. Healthy donor (HD)-derived $\mathrm{CD} 4+\mathrm{T}$ cells were co-cultured in the presence of slan+ non-classical monocytes from healthy donors $(n=2)$ or from myelodysplastic syndrome $(\mathrm{MDS})$ patients ( $\mathrm{n}=2)$. T cells at the start of the experiment (named "day 0") as well as T cells co-cultured for 5 days with slan+ non-classical monocytes were stained with a panel consisting of surface markers and intracellular markers, and markers for transcription factors and cytokines and analysed using mass cytometry (CyTOF). First, viable T cells were identified for each experiment. Then the FlowSOM algorithm was used to identify 15 metaclusters containing cells that express the same set of markers. (A) $T$ cells are visualized using viSNE plots. The expression of a selection of markers are shown in the viSNE plots for cultures containing HD- or MDS-derived slan+ monocytes at day 0 and day 5. T-cell subsets were identified based on the expression of IFN- $\gamma$, Tbet, IL-4, GATA3, IL-17, CD25, CD127, IL-10 and FoxP3 (Th1 were considered to be IFN- $\gamma$ and Tbet ${ }^{+}$IL-17 and GATA3 ${ }^{-}$; Th2 were GATA3 ${ }^{+}$or IL- $4^{+}$; Th17 were IL-17 $7^{+}$; Tregs were CD127- and FoxP3 ${ }^{+} \mathrm{CD} 25^{+}$). (B) FlowSOM-identified metaclusters were laid over day 0 and day 5 viSNE maps. Percentages of identified T-cell subsets at the start of the experiment and at day 5 are shown. Compared to day 0 , HD-derived slan+ non-classical monocytes mainly induced pro-inflammatory T cells (Th1 and Th17), as well as collateral Tregs. In contrast, T cells cultured in the presence of slan+ non-classical monocytes from MDS patients showed Th1, and above all, Th2 skewing. In HD-derived cultures Th2 cells disappeared at day 5. 
to NBM-derived cells, no statistical differences, except for IL-8 in cDC2 cultures, were found in the ability to secrete cytokines upon stimulation (Figure 5C). There was a wide variability between individual experiments indicating that cells from some MDS patients were able to secrete high amounts of cytokines, whereas others secreted hardly any. Next, isolated NBM- and MDS-derived cDC1, cDC2 and slan+ monocytes were co-cultured with HD-derived $\mathrm{T}$ cells. The capacity to induce $\mathrm{CD} 4^{+}$and $\mathrm{CD} 8^{+} \mathrm{T}$-cell proliferation was clearly reduced for all MDS-derived subsets compared to NBM-derived subsets (Figure 5D).

In order to further investigate the T-cell skewing capacity of slan+ monocytes, mass cytometry (CyTOF) was used. Both healthy PB-derived as well as MDS-derived slan+ monocytes were co-cultured with $\mathrm{CD}^{+} \mathrm{T}$ cells. Using the FlowSOM algorithm, 15 metaclusters were designated representing cells with similar marker expression profiles. Then, distinct T-cell subsets were identified and assigned to a specific metacluster using viSNE plots (Figure $6 \mathrm{~A}$ and $\mathrm{B})$. Compared to day $0 \mathrm{~T}$ cells co-cultured with HD-derived slan+ monocytes showed an increase of Th1, Th17 and Treg cells, whereas growth of Th2 cells was not supported (Figure 6B). This was in contrast with MDSderived slan+ monocytes. They induced a mixed T-cell response, including a clear Th2 cell differentiation, compared to day 0 .

\section{Discussion}

DC are important regulators of immune reactions and form a crucial bridge between the innate and adaptive immune system by directing T-cell responses. Alterations in DC frequency and function have been widely reported in the context of several diseases such as autoimmunity and cancer. ${ }^{34-38}$ In hematological malignancies the number of DC is often decreased and studies on their function mainly show an impaired induction of type-I immune responses. ${ }^{14,15,39-43}$ Slan+ monocytes have also been investigated in the context of disease. They are recruited to the site of inflammation in chronic inflammatory conditions. ${ }^{44-51}$ In cancer, including hematological malignancies, variable functional characteristics have been observed for slan+ monocytes. ${ }^{52-55}$ Enhanced stimulation of tumor-specific T-cell responses as well as differentiation into a more tolerogenic subtype have been described for this particular subset. For MDS, so far most studies that have been published describe DC in general without discriminating between subsets, or they describe in vitro generated MoDC. ${ }^{24-27,56-59}$ No reports have been published on MDSderived slan+ monocytes. Since the immune system plays an important role in MDS pathogenesis and is an attractive target for therapies, this study focused on in vivo circulating $\mathrm{cDC}$ and slan+ monocytes. Of note, in part because of their shared lineage ontogeny with MDS blasts, we decided to focus mainly on myeloid $\mathrm{cDC}$ subsets in this study and, hence, our data set lacks transcriptional and functional findings for $\mathrm{pDC}$. Moreover, at the time of this study, the recently proposed subdivision of cDC2 into DC2 and DC3 had not been recognized yet; therefore, we did not analyze discriminating markers like CD32b, CD36 and CD163. Frequencies of all studied APC subsets, except for $\mathrm{pDC}$, were lower in MDS BM compared to NBM. This decrease was most prominent in higher MDS risk groups (according to the IPSS(-R) or 2016
WHO classification). Rates of cell subsets strongly correlated between the PB and BM compartment in MDS. The finding of lower DC frequencies in MDS is important and may partly explain the poor immune responses seen in a subgroup of patients. Especially in high-risk groups, in which the dysplastic clone evades immune surveillance, restoration of the $\mathrm{CDC}$ lineage differentiation could be of benefit. Intact $\mathrm{DC}$ frequency is also relevant in vaccination strategies targeting in vivo circulating $\mathrm{cDC}$. It was shown in a phase-I trial that MDS patients with higher numbers of $\mathrm{CDC1}$ showed a more robust immune response to vaccination with the NY-ESO-1 antigen. ${ }^{60}$ Another relevant question in this context is whether lower frequencies result from clonal involvement of the DC compartment or not. Previously, Ma et al. showed clonal involvement of myeloid DC, which were characterized by the expression of CD33 and HLA-DR. ${ }^{25}$ Using the recommended phenotypic sub-division, we now clearly show that $\mathrm{CDC} 2$ are clonally involved. Unfortunately, cDC1 could not be tested because of low cell counts, but their shared lineage ontogeny with cDC2 suggests that they likely would be clonally involved as well. ${ }^{61}$ Although cell numbers were also low for slan+ non-classical monocytes, we were able to confirm mixed clonal involvement. Additional reasons for decreased frequencies of DC in MDS, such as increased apoptotic rates of hematopoietic stem cells, should be investigated in future studies. Our transcriptional profiling data showed enriched apoptotic pathways in cDC2 from MDS patients and indeed underlines this hypothesis. Very recently, Srivastava et al. showed that a decrease in DC progenitor cells could partly explain the decrease in DC frequency in MDS. ${ }^{62}$ Furthermore, they showed that higher frequencies of $\mathrm{CDC} 1$ in the $\mathrm{BM}$ of MDS patients correlated with better overall survival independent of risk categories whereas $\mathrm{CDC} 2$ frequencies did not. Reduced IRF8 expression, a crucial transcription factor for cDC1 differentiation, was associated with lower cDC1 numbers. Inhibition of LSD1, using therapeutically relevant compounds, enhanced the expression of IRF\& and subsequent differentiation to $\mathrm{CDC} 1$ and could therefore be of potential benefit in restoring DC frequencies. If this is also the case for e.g., IRF4 in cDC2 remains to be investigated.

In our transcriptional analysis of $\mathrm{cDC} 2$ and slan+ monocytes, innate immunity and danger response-related transcripts were prominently under-represented in MDSderived subsets as compared to their HD-derived counterparts. Under-represented transcripts included pattern recognition receptors, Fc- $\gamma$ receptors and down-stream signaling elements. Since these receptors and their signaling pathways form a crucial basis for normal DC/monocyte function, disruption of expression can lead to diminished immune responsiveness and possibly immune escape of dysplastic myeloid blasts and aberrant stem cells in the BM microenvironment. A wide range of TLR was found to be downregulated in MDS-derived APC. TLR are important receptors for both PAMP/DAMP-derived danger signals, which upon binding of their ligands trigger activation of downstream signaling pathways, involving, amongst others, NF-kB, MyD88 or IRAK1/4 kinases. This normally leads to the activation of pro-inflammatory transcriptional programs. Our transcriptional finding of lower PAMP/DAMP-sensing molecules and down-stream signaling genes in MDS-derived APC is therefore striking. It suggests a defective DC functionality in response to cellular 
stress. And, in the context of MDS it could lead to higher vulnerability for infections, which is often seen in this patient group. Altered TLR profiles have been observed in MDS CD34+ hematopoietic stem cells (HSC) before. ${ }^{16,63-66}$ Sustained TLR activation and constitutively activated downstream molecules as well as a loss of TLR signaling repressors have both been described in this context. Furthermore, enhanced TLR-mediated signaling in CD14 ${ }^{+}$ MDS BM cells has been shown. ${ }^{67}$ It has been suggested that together this would lead to chronic immune stimulation and subsequent DNA damage and increased cell death. In contrast, it has been shown in AML that TLR stimulation of AML blasts with TLR agonists has a positive effect and leads to differentiation. ${ }^{68}$ Thus, on one hand, increased TLR triggering can lead to excessive immune activation in hematopoietic progenitor cells, but on the other hand, decreased TLR function and defective down-stream signaling in immune effector cells (such as APC) can possibly lead to inadequate induction of immune responses and immune escape. This is important information for future studies that investigate the effect of therapeutic inhibition (by for example TLR antagonists) of these pathways in the complex MDS environment. Additionally, our gene expression data could form the basis for new research on expression levels of TLR on different MDS-derived immune cells. These expression levels can be correlated to immune cell function and therapeutic agents (e.g., TLR agonists/antagonists) should be tested in order to gain information on possible functional restoration of these cells.

DC primarily function as APC and for effective DC activity three signals are required for the interaction with $\mathrm{T}$ cells ( i) antigen presentation; ii) co-stimulation and iii) cytokine secretion). MDS BM- and NBM-derived APC were tested for their maturation, cytokine secretion and induction of T-cell priming capacity. cDC2 from MDS patients were able to upregulate HLA-DR and co-stimulatory molecules to approximately the same extent as NBM cDC2. In contrast, slan+ monocytes showed impaired maturation capacity. For both subsets, the ability for cytokine secretion seemed largely unaffected. Although the capacity of upregulating co-stimulatory molecules and secreting cytokines was intact, $\mathrm{cDC} 2$ were unable to translate this into effective T-cell proliferation induction. Also cDC1 and slan+ monocytes showed a negatively affected T-cell stimulatory function. These results are part- ly in line with previously published data on in vitro generated MoDC from MDS patients. ${ }^{26,27,56,57}$ In these studies, MoDC were already affected in their maturation and cytokine secreting capacity, underlining the difference in naturally occurring DC and MoDC. Additionally, the effect of slan+ monocytes on T-cell polarization was assessed using mass cytometry. A clear induction of proinflammatory T cells (Th1 and Th17) as well as Tregs, combined with a considerable decrease in Th2 cells was observed in cultures with HD-derived slan+ monocytes. In contrast, cultures with MDS-derived slan+ monocytes showed similar Th1/Th17 skewing, but failed to expand Tregs and, above all, Th2 cells were induced, revealing altered T-effector cell induction. Thus, in addition to lower DC frequencies, we show altered functionality of MDS APC, which again can contribute to ineffective immune responses. Of note, there is high inter-patient variability and it would be highly interesting to correlate DC function with clinical parameters (e.g., molecular background, transfusion independence, better overall and leukemia-free survival) and frequencies of other immune cells by performing extensive immune profiling studies. Interestingly, Wang et al. have suggested an inhibitory role for MDS-derived mesenchymal stem cells (MSC) that may contribute to altered MoDC function. ${ }^{59}$ Whether this is also the case for different DC subsets has to be confirmed in future research.

In conclusion, this study provides the first data on the frequency and functionality of cDC subsets and slan+ monocytes in the context of MDS. It shows a clearly affected pro-inflammatory status of MDS-derived APC which might contribute to the complex process of immune escape. A more comprehensive insight in the basal immune-pathogenesis of this disease is essential for future studies that focus on new immunotherapeutic intervention options.

\section{Disclosures}

No conflicts of interest to disclose.

\section{Contributions}

NvL-K performed experiments; NvL-K, PP, GP and JT analyzed data; NvL-K, TW, PP, GP, JT, SK, TdG, and AvdL interpreted data; NvL-K, TW, SK, TdG, and AvdL designed research; PP performed FISH experiments; NvL-K, TW, SK, PP, $T d G$ and $A v d L$ wrote the paper.

\section{References}

1. Breton G, Zheng S, Valieris R, et al. Human dendritic cells (DCs) are derived from distinct circulating precursors that are precommitted to become CD1c+ or CD141+ DCs. J Exp Med. 2016;213(13):2861-2870.

2. Breton G, Lee J, Zhou YJ, et al. Circulating precursors of human CD1c+ and CD141+ dendritic cells. J Exp Med. 2015;212(3):401413.

3. Schlitzer A, Sivakamasundari V, Chen J, et al. Identification of cDC1- and cDC2-committed DC progenitors reveals early lineage priming at the common DC progenitor stage in the bone marrow. Nat Immunol. 2015;16(7):718-728.

4. Dzionek A, Fuchs A, Schmidt P, et al. BDCA-2, BDCA-3, and BDCA-4: three markers for distinct subsets of dendritic cells in human peripheral blood. J Immunol. 2000;165(11):6037-6046.

5. MacDonald KP, Munster DJ, Clark GJ, et al. Characterization of human blood dendritic cell subsets. Blood. 2002;100(13):45124520 .

6. Robbins SH, Walzer T, Dembélé D, et al. Novel insights into the relationships between dendritic cell subsets in human and mouse revealed by genome-wide expression profiling. Genome Biol. 2008;9(1):R17.

7. Schäkel K, von Kietzell M, Hänsel A, et al. Human 6-sulfo LacNAc-expressing dendritic cells are principal producers of early interleukin-12 and are controlled by erythrocytes. Immunity. 2006;24(6):767-777.

8. Schäkel K, Mayer E, Federle C, et al. A novel dendritic cell population in human blood: one-step immunomagnetic isolation by a specific mAb (M-DC8) and in vitro priming of cytotoxic T lymphocytes. Eur J Immunol. 1998;28(12):4084-4093.

9. Schäkel K, Kannagi R, Kniep B, et al. 6Sulfo LacNAc, a novel carbohydrate modification of PSGL-1, defines an inflammatory type of human dendritic cells. Immunity. 2002;17(3):289-301

10. van Leeuwen-Kerkhoff N, Lundberg K Westers TM, et al. Human bone marrowderived myeloid dendritic cells show an immature transcriptional and functional profile compared to their peripheral blood counterparts and separate from Slan+ nonclassical monocytes. Front Immunol 2018;9(1619):1-18.

11. van Leeuwen-Kerkhoff $N$, Lundberg K, Westers TM, et al. Transcriptional profiling reveals functional dichotomy between human slan + non-classical monocytes and 
myeloid dendritic cells. J Leukoc Biol. 2017;102(4):1055-1068.

12. Hofer TP, Zawada AM, Frankenberger M, et al. slan-defined subsets of CD16-positive monocytes: impact of granulomatous inflammation and M-CSF receptor mutation. Blood. 2015;126(24):2601-2611.

13. Banchereau J, Steinman RM. Dendritic cells and the control of immunity. Nature. 1998;392(6673):245-252.

14. Mohty M, Isnardon D, Vey $\mathrm{N}$, et al. Low blood dendritic cells in chronic myeloid leukaemia patients correlates with loss of CD34+/CD38- primitive haematopoietic progenitors. Br J Haematol. 2002; 119(1):115-118.

15. Mohty, M, Jarrosay, D, Lafage-Pochitaloff $\mathrm{L}$, et al. Circulating blood dendritic cells from myeloid leukemia patients display quantitative and cytogenetic abnormalities as well as functional impairment. Blood. 2001:98(13):3750-3756.

16. Barreyro L, Chlon TM, Starczynowski DT. Chronic immune response dysregulation in MDS pathogenesis. Blood. 2018;132(15): 1553-1560

17. Epling-Burnette PK, Bai F, Painter JS, et al. Reduced natural killer (NK) function associated with high-risk myelodysplastic syndrome (MDS) and reduced expression of activating NK receptors. Blood. 2007;109 (11):4816-4824.

18. Kiladjian J-J, Bourgeois E, Lobe I, et al. Cytolytic function and survival of natural killer cells are severely altered in myelodysplastic syndromes. Leukemia. 2006; 20(3):463-470.

19. Kordasti SY, Afzali B, Lim Z, et al. IL-17producing CD4(+) T cells, pro-inflammatory cytokines and apoptosis are increased in low risk myelodysplastic syndrome. $\mathrm{Br} \mathrm{J}$ Haematol. 2009;145(1):64-72.

20. Bouchliou I, Miltiades P, Nakou E, et al. Th17 and Foxp3(+) T regulatory cell dynamics and distribution in myelodysplastic syndromes. Clin Immunol. 2011;139(3):350-359.

21. Kordasti SY, Ingram W, Hayden J, et al. CD4+CD25high Foxp3+ regulatory T cells in myelodysplastic syndrome (MDS). Blood. 2007;110(3):847-850.

22. Kotsianidis I, Bouchliou I, Nakou E, et al. Kinetics, function and bone marrow trafficking of CD4+CD25+FOXP3+ regulatory $\mathrm{T}$ cells in myelodysplastic syndromes (MDS). Leukemia. 2009;23(3):510-518.

23. Kittang $\mathrm{AO}$, Kordasti S, Sand $\mathrm{KE}$, et al. Expansion of myeloid derived suppressor cells correlates with number of $\mathrm{T}$ regulatory cells and disease progression in myelodysplastic syndrome. Oncoimmunology. 2015;5(2):e1062208.

24. Saft L, Björklund E, Berg E, et al. Bone marrow dendritic cells are reduced in patients with high-risk myelodysplastic syndromes. Leuk Res. 2013;37(3):266-273.

25. Ma L, Delforge M, van Duppen V, et al. Circulating myeloid and lymphoid precursor dendritic cells are clonally involved in myelodysplastic syndromes. Leukemia. 2004;18(9):1451-1456.

26. Ma L, Ceuppens J, Kasran A, et al. Immature and mature monocyte-derived dendritic cells in myelodysplastic syndromes of subtypes refractory anemia or refractory anemia with ringed sideroblasts display an altered cytokine profile. Leuk Res. 2007;31(10):1373-1382.

27. Matteo Rigolin G, Howard J, Buggins A, et al. Phenotypic and functional characteristics of monocyte-derived dendritic cells from patients with myelodysplastic syndromes. Br J Haematol. 1999;107(4):844850.

28. Greenberg P, Cox C, LeBeau MM, et al International scoring system for evaluating prognosis in myelodysplastic syndromes. Blood. 1997;89(6):2079-2088

29. Greenberg PL, Tuechler H, Schanz J, et al. Revised international prognostic scoring system for myelodysplastic syndromes. Blood. 2012;120(12):2454-2465

30. Kotecha N, Krutzik PO, Irish JM. Webbased analysis and publication of flow cytometry experiments. Curr Protoc Cytom. 2010;Chapter 10:Unit10.17.

31. Van Der Maaten L, Hinton G. Visualizing Data using t-SNE. J Mach Learn Res. 2008;9:2579-2605.

32. Van Gassen S, Callebaut B, Van Helden MJ, et al. FlowSOM: Using self-organizing maps for visualization and interpretation of cytometry data. Cytometry A. 2015;87(7):636-645.

33. Nizzoli G, Krietsch J, Weick A, et al. Human CD1c+ dendritic cells secrete high levels of IL-12 and potently prime cytotoxic T cell responses. Blood. 2013;122(6):932942.

34. Perrot I, Blanchard D, Freymond N, et al. Dendritic cells infiltrating human non-small cell lung cancer are blocked at immature stage. J Immunol. 2007;178(5):2763-2769.

35. Della Bella S, Gennaro M, Vaccari M, et al. Altered maturation of peripheral blood dendritic cells in patients with breast cancer. Br J Cancer. 2003;89(8):1463-1472.

36. Tran Janco JM, Lamichhane P, Karyampudi L, Knutson KL. Tumor-infiltrating dendritic cells in cancer pathogenesis. J Immunol. 2015;194(7):2985-2991.

37. Chan VS-F, Nie Y-J, Shen N, et al. Distinct roles of myeloid and plasmacytoid dendritic cells in systemic lupus erythematosus. Autoimmun Rev. 2012;11(12):890-897.

38. Carvalheiro T, Rodrigues A, Lopes A, et al. Tolerogenic versus inflammatory activity of peripheral blood monocytes and dendritic cells subpopulations in systemic lupus erythematosus. Clin Dev Immunol. 2012;2012:934161.

39. Orsini E, Calabrese E, Maggio R, et al Circulating myeloid dendritic cell directly isolated from patients with chronic myelogenous leukemia are functional and carry the bcr-abl translocation. Leuk Res. 2006;30(7):785-794.

40. Dong R, Cwynarski K, Entwistle A, et al. Dendritic cells from CML patients have altered actin organization, reduced antigen processing, and impaired migration. Blood. 2003;101(9):3560-3567.

41. Dhodapkar KM, Barbuto S, Matthews P, et al. Dendritic cells mediate the induction of polyfunctional human IL17-producing cells (Th17-1 cells) enriched in the bone marrow of patients with myeloma. Blood. 2008:112(7):2878-2885

42. Leone P, Berardi S, Frassanito MA, et al. Dendritic cells accumulate in the bone marrow of myeloma patients where they protect tumor plasma cells from CD8+ T-cell killing. Blood. 2015;126(12):1443-1451

43. Saulep-Easton D, Vincent FB, Le Page M, et al. Cytokine-driven loss of plasmacytoid dendritic cell function in chronic lymphocytic leukemia. Leukemia. 2014; 28(10):2005-2015.

44. Hänsel A, Günther C, Baran W, et al. Human 6-sulfo LacNAc (slan) dendritic cells have molecular and functional features of an important pro-inflammatory cell type in lupus erythematosus. I Autoimmun. 2013;40:1-8.

45. Hänsel A, Günther C, Ingwersen J, et al. Human slan (6-sulfo LacNAc) dendritic cells are inflammatory dermal dendritic cells in psoriasis and drive strong TH17/TH1 T-cell responses. J Allergy Clin Immunol. 2011;127(3):787-794

46. Thomas K, Dietze K, Wehner R, et al Accumulation and therapeutic modulation of 6-sulfo $\mathrm{LacNAc}(+)$ dendritic cells in multiple sclerosis. Neurol Neuroimmunol Neuroinflamm. 2014:1(3):e33.

47. Bsat M, Chapuy L, Baba N, et al. Differential accumulation and function of proinflammatory 6-sulfo LacNAc dendritic cells in lymph node and colon of Crohn's versus ulcerative colitis patients. J Leukoc Biol. 2015;98(4):671-681.

48. Ogino $T$, Nishimura J, Barman $S$, et al Increased Th17-inducing activity of CD14+ CD163 low myeloid cells in intestinal lamina propria of patients with Crohn's disease. Gastroenterology. 2013;145(6):13801391.

49. Olaru F, Döbel T, Lonsdorf AS, et al. Intracapillary immune complexes recruit and activate slan-expressing CD16+ monocytes in human lupus nephritis. JCI Insight. 2018;3(11):e96492.

50. Baran W, Oehrl S, Ahmad F, et al Phenotype, function, and mobilization of 6-sulfo LacNAc-expressing monocytes in atopic dermatitis. Front Immunol. 2018;9:1352.

51. Ahmad F, Döbel T, Schmitz M, Schäkel K Current Concepts on 6-sulfo LacNAc Expressing Monocytes (slanMo). Front Immunol. 2019;10:948.

52. Vermi W, Micheletti A, Lonardi S, et al slanDCs selectively accumulate in carcinoma-draining lymph nodes and marginate metastatic cells. Nat Commun. 2014;5 (1):3029.

53. Vermi W, Micheletti A, Finotti G, et al slan+ Monocytes and macrophages mediate CD20-dependent B-cell lymphoma elimination via $\mathrm{ADCC}$ and $\mathrm{ADCP}$. Cancer Res. 2018:78(13):3544-3559.

54. Toma M, Wehner R, Kloß A, et al. Accumulation of tolerogenic human 6-sulfo LacNAc dendritic cells in renal cell carcino$\mathrm{ma}$ is associated with poor prognosis. Oncoimmunology. 2015;4(6):e1008342

55. Lamarthée B, de Vassoigne F, Malard F, et al. Quantitative and functional alterations of 6-sulfo LacNac dendritic cells in multiple myeloma. Oncoimmunology. 2018;7(7):e1444411

56. Micheva I, Thanopoulou E, Michalopoulou $S$, et al. Defective tumor necrosis factor alpha-induced maturation of monocytederived dendritic cells in patients with myelodysplastic syndromes. Clin Immunol. 2004;113(3):31031-31037.

57. Davison GM, Novitzky N, Abdulla R. Monocyte derived dendritic cells have reduced expression of co-stimulatory molecules but are able to stimulate autologous T-cells in patients with MDS. Hematol Oncol Stem Cell Ther. 2013;6(2):49-57.

58. Micheva I, Thanopoulou E, Michalopoulou $S$, et al. Impaired generation of bone marrow CD34-derived dendritic cells with low peripheral blood subsets in patients with myelodysplastic syndrome. Br J Haematol. 2004;126(б):806-814

59. Wang Z, Tang X, Xu W, et al. The different immunoregulatory functions on dendritic cells between mesenchymal stem cells derived from bone marrow of patients with 
low-risk or high-risk myelodysplastic syndromes. PLoS One. 2013;8(3):e57470.

60. Griffiths EA, Srivastava P, Matsuzaki J, et al. Clinical NY-ESO-1 Vaccination in combination with decitabine induces antigenspecific T-lymphocyte responses in patients with myelodysplastic syndrome. Clin Cancer Res. 2018;24(5):1019-1029.

61. Srivastava P, Tzetzo SL, Gomez EC, et al. Inhibition of LSD1 in MDS progenitors restores differentiation of CD141Hi conventional dendritic cells. Leukemia. 2020;34(9):2460-2472.

62. See P, Dutertre CA, Chen J, et al. Mapping the human DC lineage through the integration of high-dimensional techniques.
Science. 2017:356(6342):eaag3009.

63. Maratheftis CI, Andreakos Moutsopoulos HM, Voulgarelis M. Tolllike receptor-4 is up-regulated in hematopoietic progenitor cells and contributes to increased apoptosis in myelodysplastic syndromes. Clin Cancer Res. 2007;13(4):1154-1160.

64. Wei Y, Dimicoli S, Bueso-Ramos C, et al Toll-like receptor alterations in myelodysplastic syndrome. Leukemia. 2013; 27(9):1832-1840

65. Dimicoli S, Wei Y, Bueso-Ramos C, et al. Overexpression of the Toll-like receptor (TLR) signaling adaptor MYD88, but lack of genetic mutation, in myelodysplastic syndromes. PLoS One. 2013:8(8):e71120.

66. Monlish DA, Bhatt ST, Schuettpelz LG. The role of Toll-like receptors in hematopoietic malignancies. Front Immunol. 2016;7:390.

67. Velegraki M, Papakonstanti E, Mavroudi I, et al. Impaired clearance of apoptotic cells leads to HMGB1 release in the bone marrow of patients with myelodysplastic syndromes and induces TLR4-mediated cytokine production. Haematologica. 2013;98(8):1206-1215.

68. Ignatz-Hoover JJ, Wang $\mathrm{H}$, Moreton SA, et al. The role of TLR8 signaling in acute myeloid leukemia differentiation. Leukemia. 2015;29(4):918-926. 